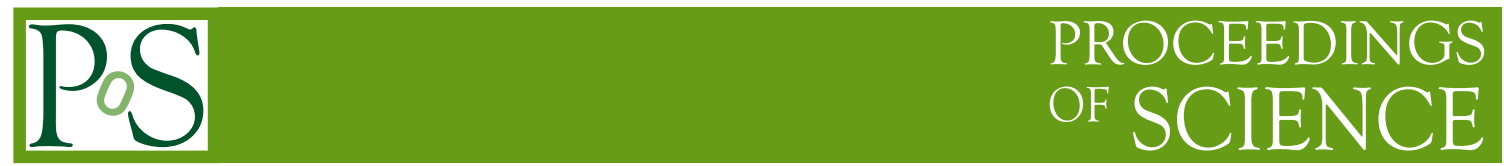

\title{
$T$ symmetry invariance tests in neutral meson decays
}

\section{Adrian BEVAN*Queen Mary University of London}

E-mail: a.j.bevan@qmul.ac.uk

We outline how the time-reversal symmetry $T$ can be systematically used to test the KobayashiMaskawa mechanism embedded in the CKM matrix using pairs of $B$ mesons created at the $\Upsilon(4 S)$ and pairs of $D$ mesons from $\psi(3770)$.

The European Physical Society Conference on High Energy Physics 18-24 July, 2013

Stockholm, Sweden

\footnotetext{
* Speaker.
} 
Weak decays are known to violate the set of improper (i.e. discrete) transformations $P$ (parity), $C$ (charge conjugation), $T$ (time-reversal), and $C P$. It is well known that electromagnetism and the strong force obey these symmetries, as bound by existing experimental data. The combination $C P T$ is related to the Lorentz group, and is conserved in locally gauge invariant quantum field theories. Models of quantum gravity may violate Lorentz invariance, and hence $C P T$. These violations in turn could manifest themselves as a difference between the $C P$ and $T$ violations found in weak decays, hence $C P, T$, and $C P T$ tests are complementary parts of a triplet of tests of quark flavour transitions. It has long been known that one can perform a Kabir asymmetry test that can be interpreted in a dual way, i.e. as a $C P$ and $T$ symmetry test [1]. In 2012 the BABAR experiment, following a prescription laid down in [2], performed a test of $T$ symmetry non-invariance (as well as $C P$ and $C P T$ tests) in $B$ decays using pairs of mesons, one decaying via a flavour filter, and the other decaying into either the $C P$-even or $C P$-odd filter state corresponding to a golden mode decay $B \rightarrow$ $c \bar{c} K_{S, L}^{0}$ [3]. These proceedings summarise the work presented in [4], where we discuss applications of this methodology to other sets of filter bases in the context of entangled pairs of neutral $B_{d, s}$ and $D$ mesons. The remainder of these proceedings start with a recap of the methodology, followed by considerations of applications to $B$ and $D$ decays before concluding with a brief summary.

Entangled pairs of neutral pseudoscalar mesons (denoted by $P_{1}$ and $P_{2}$ ) provide one with access to a physical system were the time ordering of events can be naturally reversed by comparing the two time orderings of the superposition $\Phi$. The wave function associated with such an entangled state is $\Phi=\left(\left|P_{1} P_{2}\right\rangle+\left|P_{2} P_{1}\right\rangle\right) / \sqrt{2}$, and this can be experimentally tested as there is a pair of mesons that collapse into either the first or the second time ordering. The next key point is to understand the $T$ conjugate pairs of decay filters required to experimentally reconstruct and compare between the two time orderings. In order to unambiguously test $T$ one requires two different orthonormal basis pairs. These can be any orthonormal pairs, but for convenience flavour i.e. \{particle, antiparticle $\}$ and $C P$ eigenvalue $\{+,-\}$ are obvious choices. Hence there are four distinct comparisons that can be made for a given scenario. These are (i) $\bar{P}^{0} \rightarrow P_{-}$vs $P_{-} \rightarrow \bar{P}^{0}$, (ii) $P_{+} \rightarrow P^{0}$ vs $P^{0} \rightarrow P_{+}$, (iii) $\bar{P}^{0} \rightarrow P_{+}$vs $P_{+} \rightarrow \bar{P}^{0}$, (iv) $P_{-} \rightarrow P^{0}$ vs $P^{0} \rightarrow P_{-}$, where $P^{0}\left(\bar{P}^{0}\right)$ refers to a neutral meson flavour filter decay identifying a particle or anti-particle, and those with subscripts \pm 1 refer to the eigen value of the $C P$ filter final state.

The flavour filter basis pair is defined by neutral meson decays to flavour specific final states, i.e. states that are only accessible to particle or anti-particle decays. The definition of this set could be extended to include Cabibbo allowed vs Cabibbo suppressed decays in the case of flavour tagging for $D$ mesons, at the cost of dilution of the signal. The original proposal for the $C P$ filter basis was to use the approximately orthonormal set given by the $B$ decay to a charmonium $(c \bar{c})$ plus a $K_{S}(C P=-1)$ or $K_{L}(C P=+1)$. This can be extended to include a number of other final states as discussed below. As pointed out in [4], one should also recognise that decays of pseudoscalars to two spin one (vector or axial-vector) particles also constitute a set of exact $C P$ filter basis pairs if one performs a full angular analysis to separate out the even and odd components. This broadens the range of $T$ violation tests that one can perform in the Standard Model.

Interactions resulting in the decay of $\Upsilon(4 S) \rightarrow B^{0} \bar{B}^{0}$ and $\psi(3770) \rightarrow D^{0} \bar{D}^{0}$ are equivalent in terms of quantum entanglement of the final state. This entanglement has been validated by Belle for the $\Upsilon(4 S)$ scenario [5] and is assumed to be valid for $\psi(3770)$ decays (which can be tested at a suitable charm factory). 
As detailed in [4] it is possible to measure the Unitarity Triangle angle $\beta(\alpha)$ using $b \rightarrow c, s, d$ (u) decays. In addition one can perform $T$ symmetry tests in $c \rightarrow u, d, s$ transitions at the $\psi(3770)$. For charm the goal is first to test $T$ violation in mixing, and ultimately one day to extend the interpretation to constraining the charm Unitarity Triangle angle $\beta_{c}$. Interpretation is in terms of the $T$ violating phase measured for $\lambda_{f}=(q / p)(\bar{A} / A)$. The step from mixing constraints $(q / p)$ to the weak structure of interference between mixing and decay $\left(\lambda_{f}\right)$ requires precision that goes beyond the current generation of experiments, and an improved understanding of hadronic uncertainties in the charm sector. Constraints on $\gamma$ using $c \rightarrow u$ decays are not viable as, while these processes contribute to a number of decays, they appear as sub-dominant penguin amplitudes. A review of possible interesting modes to construct $C P$ filter bases from can be found in $[4,6]$.

A number of previously unthought of $C P$ filter basis pairs can be used to search for $T$ violation. These include the following $B$ decays: the $b \rightarrow s$ loop decays $B \rightarrow\left(\eta^{\prime}, \phi, \omega\right) K_{S, L}$ and $B \rightarrow \phi K^{*} ; b \rightarrow d$ loop transitions $B \rightarrow D^{*+} D^{*-}$; the $b \rightarrow c$ transition $B \rightarrow J / \psi K^{*}$; and $B \rightarrow J / \psi \rho$ which is a colour suppressed $b \rightarrow c$ transition with a potential $b \rightarrow d$ penguin contamination, all these states measure $\beta$. Additionally one can measure $\alpha$ using $b \rightarrow u$ transitions such as $B \rightarrow \rho \rho$ and

Table 1: Estimated sensitivities on $\sigma(2 \sin 2 \beta)$ for promising $C P$ filter pairs.

\begin{tabular}{c|cc} 
Filter basis pair & $B$ Factories & Belle II \\
\hline \hline$\eta^{\prime} K_{S / L}^{0}$ & 0.6 & 0.08 \\
$\phi K^{*}$ & 1.1 & 0.13 \\
$\eta K_{S / L}^{0}$ & 1.8 & 0.17 \\
$\omega K_{S / L}^{0}$ & 2.0 & 0.22 \\
$D^{*} D^{*}$ & 2.0 & 0.29 \\
\hline
\end{tabular}
$a_{1} \rho$ decays. Table 1 summarises estimates of precisions attainable for $T$ symmetry parameters (related to $\pm 2 \sin 2 \beta$ ) for $B$ decays at the current $B$ Factories and at Belle II. Effects at the level of the Standard Model expectation should be observable in all modes at Belle II (with 50ab $\mathrm{ab}^{-1}$ of data). In terms of $D$ decays the $C P$ filter basis modes of interest include: $D^{0} \rightarrow K_{S, L}^{0}\left(\omega, \eta, \eta^{\prime}, \rho^{0}, \phi, f^{0}, a_{0}\right)$ and kinematically allowed modes with pairs of (axial-)vector mesons in the final state.

In summary we outline a set of $T$ symmetry invariance tests of $b \rightarrow u, c, d$, and $s$ as well as $c \rightarrow d$, and $s$ filter basis transitions that would enable one to over-constrain our understanding of the unitarity of the CKM matrix in terms of $B_{d, s}$ and $D$ decays in the context of the Standard Model. After almost five decades of $C P$ violation measurements it is possible to embark upon an equivalent era of $T$ (and $C P T$ ) violation tests in weak interactions to probe possible new physics contributions in tree and loop decays.

\section{References}

[1] P. K. Kabir, Phys. Rev. D 2, 540 (1970).

[2] M. C. Banuls and J. Bernabeu, Phys. Lett. B 464, 117 (1999) [hep-ph/9908353].

[3] J. P. Lees et al. [BABAR Collaboration], Phys. Rev. Lett. 109, 211801 (2012) [arXiv:1207.5832].

[4] A. Bevan, G. Inguglia and M. Zoccali, arXiv:1302.4191.

[5] A. Go et al. [Belle Collaboration], Phys. Rev. Lett. 99, 131802 (2007) [quant-ph/0702267].

[6] A. J. Bevan, G. Inguglia and B. Meadows, Phys. Rev. D 84, 114009 (2011) [arXiv:1106.5075]. 